\title{
SWOT analysis of an outpatient rehabilitation physical activity intervention for individuals with mild traumatic brain injury
}

Christophe Alarie ( $\boldsymbol{\nabla}$ christophe.alarie@umontreal.ca )

École de réadaptation, Faculté de médecine, Université de Montréal

Isabelle Gagnon

School of physical and occupational therapy, Faculty of medicine, McGill University

Lily Trang Than Huynh

École de réadaptation, Faculté de médecine, Université de Montréal

Karine Doucet

École de réadaptation, Faculté de médecine, Université de Montréal

Adèle Pichette-Auray

École de réadaptation, Faculté de médecine, Université de Montréal

Cassandre Hinse-Joly

École de réadaptation, Faculté de médecine, Université de Montréal

Bonnie Swaine

École de réadaptation, Faculté de médecine, Université de Montréal

\section{Research Article}

Keywords: Physical Activity, Exercise, Mild Traumatic Brain Injury, mTBI, Concussion, Rehabilitation, Quality improvement, Health Services, SWOT analysis

Posted Date: December 14th, 2021

DOI: https://doi.org/10.21203/rs.3.rs-1152870/v1

License: (c) (1) This work is licensed under a Creative Commons Attribution 4.0 International License. Read Full License 


\section{Abstract \\ Background}

Physical activity interventions have been shown to be an effective therapeutic approach to improve symptoms and reduce recovery time after a mild traumatic brain injury. Service providers from a specialized traumatic brain injury outpatient rehabilitation program recognized the need to ensure their physical activity intervention integrated evidence-based treatment components, while considering user needs and preferences. To inform quality improvement efforts, service providers felt it necessary to learn about the perceptions of key stakeholders, regarding the quality of the intervention. The study objective was to explore the perceptions of administrators, clinicians and users of the specialized program regarding the physical activity intervention's strengths, weaknesses, opportunities and threats (SWOT).

\section{Methods}

Using a SWOT analysis framework, this qualitative study explored the perspectives of the purposive sample $(n=14)$ composed of the managerial staff, six clinicians and five program users. Semi-structured interviews were performed, recorded, transcribed verbatim and analyzed using a qualitative content analysis approach.

\section{Results}

Fifty categories were generated resulting in 15 strengths, 17 weaknesses, 12 opportunities and 6 threats grouped into 8 overarching categories: physical activity intervention, health-related outcomes, clinical expertise, knowledge translation, communication, user engagement, resources, accessibility. Category descriptions, convergent and divergent perspectives, and salient quotes of participants are provided.

\section{Conclusions}

This study successfully identified perceived strengths, many weaknesses, several opportunities and a few threats. Participants were generally positive about the intervention but identified weaknesses including the need for service providers to better describe the physical activity intervention using theoretically driven approaches before engaging in quality improvement activities. Convergent and divergent perspectives of service providers and program users helped identify areas to maintain and others to improve upon when the program develops their new intervention. Study results may also inform the development of other physical activity interventions designed for adults with persisting symptoms of a mild traumatic brain injury.

\section{Background}


Mild traumatic brain injury ( $\mathrm{mTBI}$ ) in adults may lead to persisting symptoms and become a disabling injury for months and even years (1). Slow to recover individuals of a mTBI may have reduced body functions (e.g., cognitive limitation, exercise intolerance, emotional or sleep-wake disturbances), activity limitations (e.g., ability to walk or run), participation restrictions (e.g., work absenteeism, difficulty assuming social roles) and a reduced quality of life (2-6). Interdisciplinary management of mTBI may help reduce persisting symptoms and foster participation (7-8).

Physical activity (PA) interventions have been shown to be an effective therapeutic approach to improve symptoms and reduce recovery time post $\mathrm{MTBI}(9-10)$. Some PA interventions are based solely on progressive sub-maximal aerobic exercise, while others are more complex and include multiple modalities such as oculovestibular, coordination, or visualization exercise in addition to aerobic exercise (11-14). Although the optimal exercise parameters (e.g., length of intervention, frequency, intensity, duration, type of exercise and progression) to guide exercise prescription remain unknown $(14,15)$, PA interventions are increasingly being recommended as part of the management of adults with persisting symptoms of $\operatorname{mTBI}(7,8,16)$.

Specialized rehabilitation programs provide services to individuals with a TBI on an inpatient and outpatient basis within the public healthcare system of Quebec, Canada. A recent study found that not all of these programs offer PA interventions to their users with persisting symptoms of a mTBI (17). One particular TBI program with which our team of researchers has been collaborating was interested in reorganizing/restructuring their services before their program relocated across town. Program managers wished to evaluate the quality of certain aspects of their services, and in particular their PA intervention and teamed with researchers affiliated to their rehabilitation centre to determine how best to proceed. Recent evidence about the effectiveness of PA to reduce persistent mTBI symptoms and facilitate recovery of adults with a mTBI suggested changes to their PA intervention were needed. Since knowledge about a specific organizational context in which a change would occur is key to inform planning and executing change (18), the programs' quality improvement efforts first aimed to document stakeholders' perceptions about the quality of the existing PA intervention and of the changes needed to improve the intervention (e.g., specific exercise prescription parameters, format of delivery, goals of the intervention).

\section{Methods}

This study used a descriptive qualitative study design because the research team wanted to investigate the PA intervention in its natural state, without any attempt to manipulate or interfere with service delivery, to generate a comprehensive summary of the PA intervention as perceived and lived by individuals involved $(19,20)$. While planning and conducting the study, we used an integrated knowledge translation approach, an iterative and reflexive approach encouraging knowledge users (i.e., service providers) to be involved in production of new and tailored knowledge (21).

\section{Participants and Sampling}


Participants were service providers and program users recruited using purposive sampling to ensure information-rich cases (22). The service providers $(n=9)$ were those involved directly or indirectly in the delivery of the PA intervention at the time of the study and included the program manager, two clinical coordinators, and six clinicians (e.g., occupational therapist, social worker, physiotherapist, kinesiologist). Program users were receiving the PA intervention or had received it in the past 2 years prior to the study. Based on these criteria, a research coordinator initially contacted potential participants to determine whether they were interested in participating, and then, the first author contacted interested users to schedule an interview. Twelve program users were initially contacted by the research team and five agreed to participate.

\section{Setting}

The TBI interdisciplinary rehabilitation program team provides specialized services each year to more than 100 adults with TBI including $\approx 70$ individuals with $\mathrm{mTBI}$ living in Montréal, Québec and the surrounding area. It is comprised of a program manager, clinical coordinators, physiotherapists, occupational and speech therapists, kinesiologists, neuropsychologists and special educators. To be eligible for the rehabilitation program, users with mTBI must present with symptoms significantly impacting their participation for at least 3 months, and often have symptoms persisting for more than a year after the injury. The ultimate goals of this outpatient program are to optimize body functions and increase program users' activity and participation levels. Aspects of the PA intervention for persons with persisting symptoms of a mTBI are provided to individually or in groups primarily by physiotherapists or kinesiologists. The PA interventions aim to engage program users in low-to-moderate intensity PA by beginning with walking outdoors or low intensity water-based PA (since the facility had pool access) and progressing to more complex exercises requiring bigger muscle groups and integrating visuo-ocular components, when needed.

\section{Procedure}

To gather an important quantity of information and explore in-depth everyone's perspective, we used semi-structured interviews (22). Interview guides were developed around the SWOT analysis framework (strength [S], weakness [W], opportunity [O] and threat [T]). A SWOT analysis can help inform planning small and large reorganization of health services $(23,24)$. Perceptions about the strengths and weaknesses represent internal factors and attributes of the organization whereas the opportunities and threats represent external factors and attributes of the environment (25). Two interview guides were developed, one for the service providers and a second one, slightly modified for the program users. Guides contained one core question for each SWOT category, and associated sub-questions were used for further probing. To better tailor the interview guide toward service providers' needs, both guides were verified and enhanced for clarity by the program manager. Interviews were conducted by the first author (CA), a doctoral student and undergraduate students (CHJ, KD, APA, LTTH) external to the rehabilitation program and supervised by a senior scientist (BS). 
Before performing the semi-structured interviews, the first author familiarized himself with the program environment by performing several on-site observations, attending interdisciplinary meetings, and shadowing a kinesiologist while delivering aspects of the PA intervention. Observations were written as field notes and kept in a journal. Consent and basic demographic information were obtained from participants. Interviews were conducted in French or English at the rehabilitation centre or in a private and comfortable environment for some program users (i.e., university office). Interviews lasted less than an hour and were recorded and transcribed verbatim. To ensure the correctness of the interviews, the senior scientist (BS) listened to the recorded interviews with the first two service providers and with the first program user. Feedback to improve the quality of questioning (e.g., more emphasis on how and why) was provided. Minor adjustments were made to the interview guides (e.g., more associated questions).

\section{Analysis}

Data from all interviews were analyzed using a qualitative content analysis approach using Word software (26). During the coding phases of the analysis, service providers and program users were analyzed separately. The analysists (CA, CHJ, KD, APA, LTTH) familiarized themselves with the data by conducting multiple readings of the interview transcripts and discussed preliminary thoughts during group discussions. Relevant passages in the transcripts were coded using a 4-level coding notation based on the SWOT categories. For example, if a program user reported having trouble communicating with a service provider, the code Weakness_Communication was attributed. Each transcript was coded independently, and a second coder verified the coding correctness. Any suggested changes were resolved through group discussion under supervision of BS. When all verbatim from service providers and program users were coded, related codes were aggregated and organized in mutually exclusive categories. Using this approach, 50 categories were generated resulting in 15 strengths, 17 weaknesses, 12 opportunities and 6 threats. Further inspection of the 50 categories revealed that perceptions of service providers and users could be further grouped into 8 broader categories (see results section). To ensure confirmability of the iterative analysis process, rigorous verification of the first and co-authors' interpretation of the data by BS and by another senior scientist (IG) was performed (27). Given the small sample size and our desire to preserve anonymity, we did not specify who provided the quote when reporting citations (28).

\section{Results}

One program manager, two clinical coordinators (i.e., program administrators), six clinicians and five users were interviewed $(n=14)$. Table 1 summarizes the demographic characteristics of the program users. Regarding the service providers, two recently joined the TBI program over the last year, four had been working in the program for between 2 and 8 years, while the three managers each had over 10 years of experience with the program. They each felt they could share their perspectives about the PA intervention. 
Table 1

Demographic information of program users $(n=5)$

\begin{tabular}{|lllll|}
\hline Participant & Gender (M/F) & Age (years) & Mechanism of injury & Time since injury (mo) \\
\hline 1 & F & 33 & MVC & 28 \\
\hline 2 & F & 51 & MVC & 24 \\
\hline 3 & F & 40 & Fall & 30 \\
\hline 4 & M & 62 & Fall & 27 \\
\hline 5 & F & 51 & Fall & 13 \\
\hline Notes: M=Male Gender, F=Female Gender; MVC = Motor Vehicule Collision; mo=Month.
\end{tabular}

Table 2 summarises the eight overarching categories from the SWOT analysis: 1. PA intervention; 2. Health-related outcomes; 3. Clinical expertise; 4. Knowledge translation; 5. Communication, 6. Users' engagement, 7. Resources; 8 . Accessibility. Details and salient quotes supporting each category are presented narratively below.

[Insert Table 2 near here, please see the end of the document]

\section{PA Intervention}

The PA intervention at the time of the study was found to be accessible, individualized, and to include diverse activities such as home programming and group-based PA. To begin with, the PA intervention format (i.e., individual and group-based interventions, home-programing) and the PA intervention's flexible schedule were considered strengths by both service providers and users. Service providers felt positive about the adequateness of the PA goals given to the users and the patient-oriented approach focusing on resolving individual symptoms. Moreover, group-based interventions and home programs were considered important components by service providers and users. However, both groups identified several weaknesses such as needing more structure, specificity and clearer PA goals to enhance service quality. For instance, one service provider thought the PA intervention was less structured and "systematic" and more "random" in comparison to other interventions delivered within the specialized program (e.g., occupational therapy, neuropsychology). Similarly, some users felt the PA intervention was too general, lacked clarity with regard to targeted goals in PA and a clear exercise prescription. Interestingly, the endpoint of the intervention did not seem to be clear for users, with some users reporting that the PA intervention could be stopped before attaining rehabilitation goals. One user even reported having felt bereaved when stopping the PA intervention. Both service providers and users recognized there were opportunities to add new exercise modalities, to provide more individual or group-based direct and indirect monitoring and to improve transitioning of users into community-based organizations offering adapted PA. No threats were reported within this category.

\section{Health-related Outcomes}


The PA intervention was perceived to improve users' function and foster their return to normal activities. This overall positive impact on health-related outcomes was perceived as a strength by both groups. Indeed, they reported the PA intervention provides multiple benefits for users, such as improving their energy, mood, self-confidence, and facilitating their return to normal activities. One user reported having improved self-confidence due to the PA intervention and the close supervision of the therapist: "The fact that, specifically, I didn't have much trust in my balance, my therapist came with me outside to bicycle. This really helped me because it gave me back my confidence that I was able to do it [biking]". Users reported social benefits of the PA intervention group format in creating relationships and bonds outside of the program. Users reported creating a small peer-led support group meeting every other week outside of the program where they could share their experiences without the fear of being judged and where they did not need to justify themselves about their incapacities. No weakness, opportunity or threat was reported.

\section{Clinical Expertise}

Clinical experts' knowledge and interpersonal skills (e.g., listening, being energetic, being empathetic) were perceived to help promote the delivery of the PA intervention in a caring manner to program users. Indeed, both users and service providers thought the combination of these qualities fostered good relationships and promoted the delivery of specific PA interventions adapted to users' needs. However, when these qualities were perceived to be less present, due to a lack of expertise or experience because of staff turnover, clinical expertise was reported as a weakness of the PA intervention. For instance, users expressed the lack of empathy about the persisting symptoms of fatigue, while another user expressed the lack of ability of a clinician to motivate them. Interestingly, some characteristics of the users such as their beliefs, physical and intellectual limitations, occupations, or symptoms are considered threats to the PA intervention by both service providers and users. For example, users may have concomitant injuries, post-traumatic stress disorders or experience kinesiophobia needing particular attention: "Sometimes, it depends on their injury. If they have a musculoskeletal injury, it may limit [the intervention]. So we have this to consider. Or, also, if they have post-traumatic stress disorder sometimes or kinesiophobia. This can alter [the intervention], in the sense that some are afraid of movement...". The clinicians would then have to work around users' characteristics to offer an adapted PA intervention considered less beneficial than if the intervention was not adapted. No opportunities were reported.

\section{Knowledge Translation}

Translating knowledge about PA for individuals with a mTBI was believed to have an impact on service quality and accessibility to the program. Indeed, service providers felt their rehabilitation center had challenges translating evidence-based knowledge about PA for individuals with a mTBI within their program and difficulty promoting their clinical expertise outside the rehabilitation center. Moreover, they considered the lack of time for professional development or quality improvement efforts as a weakness that hindered PA intervention delivery. For instance, one service provider reported that "It is hard to free all clinicians' schedule for one afternoon and think about how to organise this component [PA intervention] of the program. It's the users that are not being seen during this time". Service providers felt they should 
develop strategies to more efficiently translate evidence-based knowledge to their clinicians to enrich their clinical expertise and improve PA intervention delivery. Similarly, fostering collaboration with researchers within the institution and/or with specialized service providers outside of the program could enhance knowledge-sharing capacity and ultimately improve the quality of the PA intervention and accessibility to the program. Another perceived opportunity included sharing the program's expertise about PA and mTBI with physicians outside of the program and other local rehabilitation centres to enhance program referral. There was also a shared perception about the lack of knowledge among external organizations (e.g., insurance companies, physicians) about the health-related effects of $\mathrm{mTBI}$ and the specialized management required to treat mTBI. This lack of knowledge is thought to be responsible for low numbers of referrals to the program and therefore reduced access to the PA intervention. No strengths were reported for this category.

\section{Communication}

Service providers reported working in interdisciplinarity to elaborate common treatment goals and to communicate a common message about PA to program users. Communication between service providers in this manner was generally perceived as a strength by service providers and some users. Service providers also reported their physical work environment (i.e., offices located near each other) and technological communication tools fostered good formal and informal communication. Despite the overall perception of good communication between the service providers, two main communication issues (weaknesses) were reported. First, the overlap of professional roles of service providers from different disciplines (e.g., physiotherapy and kinesiology) created tension between colleagues and disagreements. Second, contrary to the perception of the team's capacity to elaborate and communicate a clear message about PA to their users, conflicting messages communicated to users was mentioned. One user reported being frustrated by a miscommunication about the termination of PA intervention when he thought he was continuing the services: "I think it is terrible! And then they told me: "Well, she planned to end the [PA] intervention with you." Well... she hasn't told me that, she even said that we would probably try to do some biking outside soon". No opportunities or threats were reported.

\section{Users' Engagement}

Service providers and users reported wanting to improve user's engagement in the PA intervention. Both groups saw several opportunities to improve responsibility sharing and shared decision-making as means to improve users' engagement. For example, service providers suggested that all program users should receive general information about PA for individuals with a mTBI using a small group format to ensure they all receive the same information before starting the PA intervention. Furthermore, one service provider suggested creating a detailed contract with each user to foster adherence to the PA intervention. Finally, both service providers and users thought users should receive more information about the "risks" associated with being involved in group-based PA performed at the rehabilitation center (e.g., falls, body collisions, head impacts). One user suggested written informed consent could be obtained by users to demonstrate their understanding and commitment to this PA intervention modality. No strength, weakness or threats was reported. 


\section{Resources}

Service providers and users reported that adequate human, financial and material resources are crucial to support the PA intervention delivery. Both groups reported that the infrastructure, including the swimming pool, the exercise room and its equipment, and the adapted break room for relaxation, was a great strength of the program. It allows service providers to offer varied, adapted, and appropriate interventions. However, service providers and program users shared several weaknesses about available resources. To begin, some service providers and users indicated that treatment options were limited because of restricted access to the swimming pool. One user reported feeling at risk of an injury by using inadequate exercise equipment: "There is one stationary bike that I was using at the beginning that had an electrical resistance, then at one point, it stopped working. I was pedalling without any resistance. So it puts you at risk for an injury". In the same line of thought, users reported not having adequate equipment at home to perform prescribed exercises, which may hinder their adherence to the PA intervention. Service providers and users shared the perception that staff turnover, reduced staff and lack of job stability were threats to the PA intervention. A service provider felt the intervention was vulnerable to staff changes: "Each time we change personnel, each time we are losing expertise. So that is a threat for the physical activity services particularly". Budget restrictions were also perceived as a threat by service providers directly involved in the PA intervention: "Yeah... If there is less budget, well it's possible that they cut (days of work) in certain professions such as mine or kinesiology (...)". The upcoming relocation of the specialized program was perceived as a threat to the PA intervention because of potential loss of access to adapted gymnasiums, equipment and spaces all used to support the PA intervention. No opportunities were reported.

\section{Accessibility}

Service providers and users both highlighted the importance of having access to a well-located and easily accessible rehabilitation centre. The geographical location of the rehabilitation center at the time of the study was considered a strength as it allows community-based PA and real-life scenario interventions. For example, one service provider reported the ease of delivering PA intervention outside in parks around the rehabilitation center. However, the relocation of the TBI program to a different rehabilitation hospital in another part of the city was perceived as threatening the accessibility to the TBI program and consequently, to the PA intervention. Service providers were uncertain/worried about the ease of user access to the new location (e.g., less available parking, reduced public transportation, further from the current location's catchment area) and the available environment for their PA intervention (e.g., swimming pool, parks). Users also reported being worried the relocation would negatively impact the accessibility of program services. One user reported believing the relocation might force some current or future users to be less adherent to treatments or to abandon them: "Well, there will be more and more people that will just abandon, or they won't go to their treatments". No weakness or opportunities were reported.

\section{Discussion}


This study aimed to explore the perceptions of service providers and users about the strengths, weaknesses, opportunities and threats of a PA intervention designed for individuals with a mTBI delivered within a specialized TBI rehabilitation program. This study successfully identified perceived strengths, identified many weaknesses, several opportunities, and a few threats about the current PA intervention, suggesting a positive context for change and quality improvement efforts.

A main result of this study was identifying that important characteristics about the current PA intervention were not clearly understood by service providers and program users. Specifically, it appears the therapeutic goals of the PA intervention and the exercise parameters are individualized and adapted to user-specific needs, which may explain the perceived "random" and "variable" nature of the goals and exercise prescription. Moreover, clinical managers and researchers identified at the outset of this project that the PA intervention was not formally documented, and exercise prescription parameters were not defined. This most probably contributed to this perception, since without clear goals and a replicable intervention program users may not receive an adequate PA intervention. This method of delivering interventions adapted to users' needs and ability are in line with values of patient-centred care (29). However, a patient-centred care approach should not prevent an organization from knowing the goals and the processes required to attain these goals. Before assessing the quality or initiating quality improvement efforts of a health intervention, it is important to clearly establish valid relationships between structure, process and outcomes of an intervention (30).

Despite lack of clarity about what is being delivered to program users, the PA intervention is thought to improve some health-related outcomes. These perceived benefits align with literature supporting the effectiveness of PA on mood and return to normal activities and add to an emerging literature supporting improvement of self-esteem following mild TBI (11,31-36). Interestingly, neither service providers nor program users reported the PA intervention as a means to reduce persisting symptoms of a mTBI although recent systematic reviews and meta-analyses indicate that PA, and more precisely cardiovascular exercise, is effective in reducing persisting symptoms of a mTBI and hastens return to normal activity (10). There are multiple ways of interpreting these results. It is plausible service providers have different beliefs, are unaware, or are not comfortable with this new evidence-based approach for this population, or they may have distinct rehabilitation goals other than persisting symptoms reduction. Existing knowledge translation challenges may explain why targeted health-related outcomes of the PA intervention are distinct from what is most reported in literature.

Interestingly, an unexpected outcome of the PA intervention identified in this study was the creation of a peer-led support group by users participating in group-based PA activities. This may demonstrate the positive impacts of group dynamics, but more importantly, further highlight the social needs of adults with persistent symptoms of $\mathrm{mTBI}$ to socialize and share experiences among peers (37). Literature about the potential benefits of peer-led support groups for individuals with mTBI is scant, however, there is growing evidence about how peer-led programs may provide multiple social benefits and improve healthrelated outcomes among individuals with moderate and severe TBI, stroke and spinal cord injury (38-40). Service providers may want to explore including a peer-support aspect in their PA intervention. 
An important upcoming challenge reported by service providers is maintaining the PA intervention accessible after the specialized program relocates to a rehabilitation hospital beyond the current catchment area. Indeed, the geographical location of a health service is a well-known barrier to healthcare accessibility and may lead to a diverse set of negative consequences (e.g., physical, psychological, social, and economic consequences) if individuals have reduced access to health services $(41,42)$. Users included in this study feared the move could lead users to abandon their interventions. As users reported appreciating the home-based PA component of the PA intervention, service providers could offer enhanced home programs or explore offering adjunct telerehabilitation services to increase or maintain adherence. This suggestion is relevant considering the current COVID-19 pandemic.

Besides being the first study to explore the perceptions about the quality of a PA intervention for persons with persisting symptoms of a $\mathrm{mTBl}$, an important contribution of this study was combining the unique perspectives of service providers with those of program users in this SWOT analysis. Most studies reporting results of a SWOT analysis in healthcare only include the perspective of providers $(23,24)$ or patient organisations (43). Although there were convergent opinions about the general appreciation of some intervention characteristics (e.g., accessible, individualized, group-based), divergent opinions were reported about aspects of the PA intervention (e.g., adequateness of the PA intervention, clarity of goals, exercise prescription, home program feasibility, miscommunication between clinical experts). These results are in line with prior research in quality of care, highlighting the importance of integrating patient perspective $(44,45)$.

\section{Recommendations}

This study was conducted to better understand an existing PA intervention to guide future quality improvement efforts. Indeed, implementation of change often fails because of barriers at multiple levels within a health organization (e.g., patient level, provider team level, organizational or the policy level) (46). Understanding of the inner workings and outer setting of a health organization helps to identify potential barriers, can guide selection of effective interventions and ultimately, improve strategical planning of quality improvements. This study helped identify areas of the current PA intervention to maintain and others that could be improved upon. Two main recommendations stem from the study results. First, to enhance and evaluate their current services, there is a need to further describe the underlying intervention theory of the existing PA intervention, its processes and its goals (47). To help describe the PA intervention and plan quality improvement efforts, service providers could build a logic model, a visual representation of the relationships between resources, process and outcomes of a health intervention (47). Mobilizing enough time and resources to generate a logic model of the PA intervention may be a challenging endeavour for a clinical program that reports time constraints for knowledge translation and quality improvement efforts. However, besides ensuring a standardized and replicable intervention for all users, service providers would then be able to compare their PA intervention to most the recent evidence and thus make appropriate choices about evidenced-based approaches to implement. 
The second recommendation aims to improve the knowledge translation capacity of the specialized program. Identifying solutions to overcome this barrier, such as embedding quality improvement activities in normal clinical workflow (18) or obtaining funding to support partial release of clinical work of key clinical experts, could be envisaged. Moreover, involving professionals with research and communication skills such as knowledge brokers and/or maintaining research and quality improvement activities with researchers could help improve service quality and promote evidence-based practice $(18,48)$.

\section{Study Limitations}

The results of this study may have limited transferability to other TBI rehabilitation programs as they are based on the perspectives about a local program and intervention. However, to our knowledge few PA interventions for persons with persisting symptoms have been described in the literature. As such, the results could assist other programs in the process of creating or developing PA interventions for adults with persisting symptoms of a mTBI. Saturation may not have been attained because of the limited number of study participants. Also, a social desirability bias is possible; both users and service providers may have presented a more favourable picture of the PA intervention. However, the presence of conflicting and somewhat negative perceptions of the PA intervention indicates at least some of the participants shared their critical opinions about the PA intervention, thus contributing important information to quality improvement efforts.

\section{Conclusion}

This study supports the use of SWOT analysis as a promising tool to uncover the perceptions of key stakeholders for quality improvement. By openly sharing their perspective and thoughts about the current PA intervention, both service providers and program users showed their willingness to improve the quality of services. This study successfully identified strengths, weaknesses, opportunities and threats related about the quality of a PA intervention within a specialized TBI rehabilitation program and thus provides key insights about components of the PA intervention to maintain or to build upon during upcoming quality improvement efforts. Further description of the current PA intervention using a theoretically driven program evaluation tool (i.e., a logic model) is warranted before initiating quality improvement effort.

\section{Abbreviations}

PA

Physical activity

TBI

Traumatic brain injury

mTBI

mild traumatic brain injury

SWOT

Strength, weakness, opportunity, and threat 


\section{Declarations}

\section{Ethics approval and consent to participate:}

The study received approval from the Centre for interdisciplinary research in rehabilitation of the greater Montreal's Research ethics board (CRIR1273-1017). Written consent was obtained for service providers and program users.

\section{Consent for publication:}

Not applicable.

\section{Availability of data and materials:}

The data that support the findings of this study are available from the corresponding author (CA) on reasonable request. The data are not publicly available due to containing information that could compromise research participant privacy/consent.

\section{Competing interests:}

The authors declare that they have no competing interests.

\section{Funding}

This research has been supported, in part, by the Fonds de Recherche du Québec en Santé (FRQS). CA received a bursary of Excellence from the École de réadaptation of the Université de Montréal.

\section{Authors' contributions}

CA, BS and IG designed the study, CA, BS, IG, CHJ, KD, APA, LTTH, collected, analyzed and interpreted the results. CA wrote the draft of the manuscript and all authors reviewed and approved the final manuscript.

\section{Acknowledgements:}

We thank our clinical partners and the program users of the specialized TBI program of the Centre Universitaire Intégré en Santé et Services Sociaux du Centre-Sud-de-l'île-de-Montréal for their involvement in this study.

\section{References}

1. Cassidy JD, Cancelliere C, Carroll LJ, Côté P, Hincapié CA, Holm LW, et al. Systematic review of selfreported prognosis in adults after mild traumatic brain injury: results of the International Collaboration on Mild Traumatic Brain Injury Prognosis. Arch Phys Med Rehabil. 2014;95(3 Suppl):S132-51. 
2. Cooksley R, Maguire E, Lannin NA, Unsworth CA, Farquhar M, Galea C, et al. Persistent symptoms and activity changes three months after mild traumatic brain injury. Aust Occup Ther J. 2018;65(3):168-75.

3. Fineblit S, Selci E, Loewen H, Ellis M, Russell K. Health-related quality of life after pediatric mild traumatic brain injury/concussion: a systematic review. J Neurotrauma. 2016;33(17):1561-8.

4. Voormolen DC, Polinder S, Von Steinbuechel N, Vos PE, Cnossen MC, Haagsma JA. The association between post-concussion symptoms and health-related quality of life in patients with mild traumatic brain injury. Injury. 2019;50(5):1068-74.

5. Ouellet M-C, Beaulieu-Bonneau S, Morin CM. Sleep-wake disturbances after traumatic brain injury. Lancet Neurol. 2015;14(7):746-57.

6. Kozlowski KF, Graham J, Leddy JJ, Devinney-Boymel L, Willer BS. Exercise intolerance in individuals with postconcussion syndrome. J Athl Train. 2013;48(5):627-35.

7. Ontario Neurotrauma Foundation (ONF). Guideline for concussion/mild traumatic brain injury \& persistent symptoms 3rd edition, for adults over 18 years of age. Toronto: Ontario Neurotrauma Fondation (ONF); 2018. http://braininjuryguidelines.org/concussion/index.php?id=1.

8. McCrory P, Meeuwisse W, Dvorak J, Aubry M, Bailes J, Broglio S, et al. Consensus statement on concussion in sport-the 5th international conference on concussion in sport held in Berlin, October 2016. Br J Sports Med. 2017;(11):838-47.

9. Lal A, Kolakowsky-Hayner SA, Ghajar J, Balamane M. The Effect of Physical Exercise After a Concussion: A Systematic Review and Meta-analysis. Am J Sports Med. 2018;46(3):743-52.

10. Langevin P, Frémont P, Fait P, Dubé M-O, Bertrand-Charette M, Roy J-S. Aerobic Exercise for Sportrelated Concussion: A Systematic Review and Meta-analysis. Med Sci Sports Exerc. 2020;52(12):2491-99.

11. Kleffelgaard I, Soberg HL, Tamber A-L, Bruusgaard KA, Pripp AH, Sandhaug M, et al. The effects of vestibular rehabilitation on dizziness and balance problems in patients after traumatic brain injury: a randomized controlled trial. Clin Rehabil. 2019;33(1):74-84.

12. Gagnon I, Grilli L, Friedman D, Iverson GL. A pilot study of active rehabilitation for adolescents who are slow to recover from sport-related concussion. Scand J Med Sci Sports. 2016;26(3):299-306.

13. Leddy JJ, Haider MN, Ellis MJ, Mannix R, Darling SR, Freitas MS, et al. Early subthreshold aerobic exercise for sport-related concussion: a randomized clinical trial. JAMA pediatrics. 2019;173(4):31925.

14. Alarie C, Gagnon I, Quilico E, Teel E, Swaine B. Physical Activity Interventions for Individuals With a Mild Traumatic Brain Injury: A Scoping Review. 2021;01:36(3)205-223.

15. Howell DR, Taylor JA, Tan CO, Orr R, Meehan WP. The Role of Aerobic Exercise in Reducing Persistent Sport-related Concussion Symptoms. Med Sci Sports Exerc. 2019;51(4):647-52.

16. Department of Defense and Veterans Affairs (DoD/VA). VA/DoD clinical practice guideline for management of concussion/mild traumatic brain injury. Washington; 2016. p. 53. 
17. Alarie C, Gagnon I, Gagnon S, Gendron D, Girard C, Swaine B. Physical activity interventions in rehabilitation programs for outpatients with mild traumatic brain injury. Res Q Exerc Sport. (Forthcoming).

18. Batalden PB, Davidoff F. What is "quality improvement" and how can it transform healthcare?: Qual Saf Health Care. 2007;16(1):2-3.

19. Beuving J, De Vries G. Doing qualitative research: the craft of naturalistic inquiry. Amsterdam: Amsterdam University Press; 2015.

20. Colorafi KJ, Evans B. Qualitative descriptive methods in health science research. HERD. 2016;9(4):16-25.

21. Bowen SJ, Graham ID. From Knowledge Translation to Engaged Scholarship: Promoting Research Relevance and Utilization. Arch Phys Med Rehabil. 2013;94(1, Supplement):S3-S8.

22. Martinson K, O'Brien C. Conducting case studies. In: Newcomer KE, Hatry HP, Wholer, JS. Handbook of practical program evaluation. Jossey-Bass. New Jersey;2015:177-96.

23. Camden C, Swaine B, Tétreault S, Bergeron S. SWOT analysis of a pediatric rehabilitation programme: A participatory evaluation fostering quality improvement. Disabil. 2009;31(16):137381.

24. van Wijngaarden JD, Scholten GR, van Wijk KP. Strategic analysis for health care organizations: the suitability of the SWOT-analysis. Int J Health Plann Manage. 2012;27(1):34-49.

25. Gürel E, Tat M. Swot Analysis: A Theoretical Review. Int. J. Soc. Res. Methodol. 2017;10(51):9941006.

26. Vaismoradi M, Turunen H, Bondas T. Content analysis and thematic analysis: Implications for conducting a qualitative descriptive study. Nurs Health Sci. 2013;15(3):398-405.

27. Miles MB, Huberman AM, Saldaña J. Qualitative data analysis: A methods sourcebook. 3rd. Thousand Oaks, CA: Sage; 2014.

28. Saunders B, Kitzinger J, Kitzinger C. Anonymising interview data: challenges and compromise in practice. Qual Res. 2015;15(5):616-32.

29. Epstein RM, Street RL. The values and value of patient-centered care. Annals Family Med. 2011;9(2):100-3.

30. Donabedian A. The quality of care: how can it be assessed? JAMA. 1988;260(12):1743-8.

31. Kleffelgaard I, Soberg HL, Bruusgaard KA, Tamber AL, Langhammer B. Vestibular Rehabilitation After Traumatic Brain Injury: Case Series. Phys Ther. 2016;96(6):839-49.

32. Gemmell C, Leathem JM. A study investigating the effects of Tai Chi Chuan: Individuals with traumatic brain injury compared to controls. Brain Inj. 2006;20(2):151-6.

33. Leddy JJ, Kozlowski K, Donnelly JP, Pendergast DR, Epstein LH, Willer B. A preliminary study of subsymptom threshold exercise training for refractory post-concussion syndrome. Clin J Sport Med. 2010;20(1):21-7. 
34. Adams J, Moore B. Return to meaningful activities after a multi-modal rehabilitation programme among individuals who experience persistent dizziness and debility longer than 9 months after sustaining a concussion: A case series. Physiother Can. 2017;69(3):249-59.

35. Yost TL, Taylor. Qigong as a novel intervention for service members with mild traumatic brain injury. Explore (NY). 2013;9(3):142-9.

36. Lawrence DW, Richards D, Comper P, Hutchison MG. Earlier time to aerobic exercise is associated with faster recovery following acute sport concussion. PLOS One. 2018;13(4):e0196062.

37. Bannon SM, Greenberg J, Goldson J, O'Leary D, Vranceanu A-M. A social blow: the role of interpersonal relationships in mild traumatic brain injury (mTBI). Psychosom. 2020(61):518-26.

38. Hughes R, Fleming P, Henshall L. Peer support groups after acquired brain injury: a systematic review. Brain Inj. 2020;34(7):847-56.

39. Sweet SN, Michalovic E, Latimer-Cheung AE, Fortier M, Noreau L, Zelaya W, et al. Spinal cord injury peer mentorship: applying self-determination theory to explain quality of life and participation. Arch Phys Med Rehabil. 2018;99(3):468-76. e12.

40. Barclay L, Hilton GM. A scoping review of peer-led interventions following spinal cord injury. Spinal Cord. 2019;57(8):626-35.

41. Neri MT, Kroll T. Understanding the consequences of access barriers to health care: experiences of adults with disabilities. Disabil. 2009;25(2):85-96.

42. Veltman A, Stewart DE, Tardif GS, Branigan M. Perceptions of Primary Health Services Among People with Physical Disabilities. Part1: access issues. MedGenMed. 2001;3(2):18.

43. Van Durme T, Macq J, Anthierens S, Symons L, Schmitz O, Paulus D, et al. Stakeholders' perception on the organization of chronic care: a SWOT analysis to draft avenues for health care reforms. BMC Health Serv. Res. 2014;14(1):1-9.

44. Gleeson H, Calderon A, Swami V, Deighton J, Wolpert M, Edbrooke-Childs J. Systematic review of approaches to using patient experience data for quality improvement in healthcare settings. BMJ open. 2016;6(8):e011907.

45. Pomey M-P, Ghadiri DP, Karazivan P, Fernandez N, Clavel N. Patients as partners: a qualitative study of patients' engagement in their health care. PloS one. 2015;10(4):e0122499.

46. Damschroder LJ, Aron DC, Keith RE, Kirsh SR, Alexander JA, Lowery JC. Fostering implementation of health services research findings into practice: a consolidated framework for advancing implementation science. Implement. 2009;4(1):50.

47. Champagne F, Brousselle A, Hartz Z, Contandriopoulos A, Denis J. Modéliser les interventions. In: L'évaluation: concepts et méthodes: Deuxième Édition. 2 ed. Montréal: Les presses de l'Université de Montréal; 2011. p. 57-70.

48. Gaid D, Ahmed S, Alhasani R, Thomas A, Bussières A. Determinants that influence knowledge brokers' and opinion leaders' role to close knowledge practice gaps in rehabilitation: A realist review.J Eval Clin Pract. 2021;27(4):836-46. 


\section{Table 2}

TABLE II: Overarching categories emerging from strengths, weaknesses, opportunities and threats perceived by service providers and users of the TBI specialized program $(n=14)$ 


$\begin{array}{ll}\text { Overarching } & \begin{array}{l}\text { Description of overarching category and } \\ \text { category }\end{array}\end{array}$

PA intervention The PA intervention is accessible, individualized, and includes diverse activities such as home programming and group-based activity, but needs more structure, specificity and clear goals to enhance service quality

- PA intervention goals and exercise sessions

\section{$S$} are individualized and adaptable to an individual's symptom status

- Group information sessions about resuming PA with $\mathrm{mTBI}$ and group-based adapted PA such as dance therapy and adapted collective sports offered in the rehabilitation center, provide socialisation opportunities and foster interactions between users

- Home programming is appreciated by users

S and allows them to stay active at home

- Access to clinicians responsible for PA

S $\quad \checkmark \quad r$

$\checkmark \quad \checkmark$
intervention is easy and offers global management of $\mathrm{mTBI}$

- PA session format and flexible scheduling are S adequate

- PA intervention is not structured and is lacking home follow-ups

- Clinical experts' schedules are limited,

W

- PA intervention is general and is not adapted to user's capacity and needs

- PA intervention goals and the exercise prescribed are complex, unclear and not understood by users 
- PA intervention may be stopped before reaching rehabilitation goals

- Stopping PA intervention can create bereavement feelings for users

- There are delays for users to obtain interdisciplinary treatments

- Adding new activities such as relaxation, yoga and swimming could complement and improve the PA intervention

- Provide more opportunities for direct or 0 0 $\checkmark$ $\checkmark$ indirect monitoring such as maintaining access to sports facilities during user transition periods upon arrival, during and at the end of the program

- Offer more group and individual exercise

0 $\checkmark \quad \checkmark$ sessions before, during and after interdisciplinary interventions

- Transfer users at the end of the PA 0 $\checkmark$ intervention to community organizations specializing in adapted PA

- All users should have access to kinesiology

0 and the PA intervention

PA intervention improves users' function and participation

- PA intervention allows users to surpass themselves, to improve health-related outcomes such as self-confidence and mood, in addition to facilitating return to normal daily activities

- Users having participated in group intervention sessions created a peer-led support group which frequently met outside of the program 


\section{Clinical expertise}

- Clinicians' expertise allows offering specific

$S$

PA interventions adapted to users' needs and capacities

- Clinicians' interpersonal competences such as

S

listening, being energetic, and empathetic contribute to providing humanist care and foster good relationships

- Staff turnover may create situations where W $\checkmark$ clinicians responsible for the PA intervention have less experience to provide evidencebased treatment

- Some clinicians are not motivating and lack empathy about persisting symptoms

- The beliefs, physical and intellectual limitations, occupations, and symptoms of users can interfere with the performance of the PA intervention
Knowledge translation
Knowledge translation about PA for individual with a $\mathrm{mTBI}$ enhances services quality

- Lack of time for professional development W $\quad \checkmark$ and program quality improvement efforts

- Developing processes to transfer evidencebased knowledge to clinicians would enrich their knowledge and improve PA intervention delivery

- Collaborating with researchers and clinicians specialized in oculovestibular therapy outside of the program could enhance PA intervention quality

- Sharing TBI specialized expertise in PA with 0

$0 \quad \checkmark$
physicians and other rehabilitation centers locally 
- Lack of knowledge about health-related effects of mTBI and its management by external organizations (e.g., insurance companies, physicians) reduces referrals to program and access to PA intervention

Communication Communication between clinicians and users ensures interdisciplinarity services and contributes to communicating a single vision about PA to users

- Clinicians work in interdisciplinary teams

S $\checkmark \checkmark v$
allowing them to elaborate common treatment goals and communicate a single vision about PA

S $\quad \checkmark$

- Clinicians' offices are strategically situated to facilitate interprofessional communication and PA intervention

- Clinicians have access to various tools S $\quad \checkmark$ fostering interprofessional communication

- Administrative agent offers critical support to S clinicians for coordinating services

- Overlaps in professional roles of clinicians W responsible of PA intervention generate disagreements

- Clinicians lack communication among themselves which provides users conflicting messages about PA

Users' engagement
Engaging program users in decision-making and responsibility sharing may improve PA participation

- Users could receive more information about possible risks of group-based adapted PA and consent to participate

- Creating a contract with users before 
admission to the program could consolidate their commitment to the PA intervention

- Mandatory participation in an information group when users are admitted to the program could ensure all users receive the same information about PA before starting treatments

- Possibility of creating a peer-led support group that is not supervised by a clinician

- Infrastructures such as the pool, the exercise room and the adapted break room, allow clinicians to offer varied, adapted and adequate PA interventions to users

- Exercise room is not adapted to deliver safe W

$\checkmark \quad \checkmark$
and efficient PA intervention

- Access to the swimming pool is limited, W $\checkmark$ decreasing treatment options for clinicians

- Lack of personnel impairs PA intervention W $\checkmark$ delivery to users

- Some exercise equipment needs maintenance W or replacement, limiting PA interventions

- User's home equipment may not be adequate W to perform at-home prescribed exercise

- Job insecurity, lack of human resources and T staff turnover can undermine the expertise of the program and affect PA intervention delivery

- Budget restriction can affect the PA intervention delivery

- Gymnasium, some equipment and spaces 
useful for PA intervention will be lost after the relocation

Accessibility Users have access to a well-located rehabilitation center allowing community-based PA and real-life scenario interventions

- Rehabilitation center's geographical location

S increases accessibility, and it is well situated to provide community-based PA, and real-life scenario interventions

- Upcoming relocation of the specialized program generates uncertainty about accessibility to PA intervention

Notes: PA = Physical Activity; $\mathrm{mTBI}=$ Mild Traumatic Brain Injury; SWOT= Strength, Weakness, Opportunity, and Threat; $\mathrm{S}=$ Strength, $\mathrm{W}=$ Weakness, $\mathrm{O}=$ Opportunity, $\mathrm{T}=$ Threat; $\checkmark=$ Yes. 